\title{
Appetite during consumption of enteral formula as a sole source of nutrition: the effect of supplementing pea-fibre and fructo-oligosaccharides
}

\author{
Kevin Whelan ${ }^{1}$, Loukia Efthymiou ${ }^{1}$, Patricia A. Judd ${ }^{2}$, Victor R. Preedy ${ }^{1}$ and Moira A. Taylor ${ }^{3}$ \\ ${ }^{1}$ Nutritional Sciences Research Division, King's College London, 150 Stamford Street, London SE1 9NH, UK \\ ${ }^{2}$ Lancashire School of Health and Postgraduate Medicine, Harrington Building, University of Central Lancashire, \\ Preston PRI 2HE, UK \\ ${ }^{3}$ School of Biomedical Sciences, Medical School, University of Nottingham, Nottingham NG7 2UH, UK
}

(Received 22 July 2005 - Revised 21 February 2006 - Accepted 27 February 2006)

\begin{abstract}
Liquid enteral formulas are commonly used as a sole source of nutritional support of patients in hospital and community settings. Their effect on appetite has important consequences for dietary management of such patients and is likely to be affected by the formula composition. The aim of the present study was to compare appetite within healthy subjects consuming both a standard formula and one supplemented with pea-fibre (10 g/l) and fructo-oligosaccharide (FOS; $5 \mathrm{~g} / \mathrm{l}$ ) as a sole source of nutrition. Eleven healthy subjects consumed a standard formula or a pea-fibre/FOS formula as a sole source of nutrition for $14 \mathrm{~d}$ in a double-blind, cross-over trial. Appetite was recorded using standard $100 \mathrm{~mm}$ lines anchored at each end by a phrase denoting the most extreme appetite sensation. Consumption of the pea-fibre/FOS formula resulted in higher mean fullness (46 v. $37 \mathrm{~mm}, P=0.035)$, minimum fullness $(13 v .9 \mathrm{~mm}, P=0.024)$ and minimum satiety $(12 v .8 \mathrm{~mm}, P=0.012)$ compared to the standard formula. As there were no differences in macronutrient intake between formulas, these differences are likely to be due to supplementation with peafibre and FOS. The effect on appetite of the composition of an enteral formula, both with respect to nutrient content and functional components such as pea-fibre and FOS, may be an important aspect to consider in the dietary management of patients consuming enteral formula as a sole source of nutrition.
\end{abstract}

Enteral nutrition: Appetite: Fibre: Fructo-oligosaccharides: Hunger: Prebiotics

Liquid enteral formulas are commonly used in both hospital and community settings for the nutritional support of patients who are unable to achieve their nutritional requirements from food alone. Enteral formulas can be used to supplement an inadequate oral diet (e.g. anorexia, hypermetabolism, undernutrition) or as a sole source of nutrition, either because the patient is unable to eat (e.g. unconscious, dysphagic) or because they are required to abstain from eating food (e.g. Crohn's disease) (Pearce \& Duncan, 2002). Enteral formulas are either delivered orally or via a tube directly into the gastrointestinal tract (enteral tube feeding (ETF)), depending upon the patient's ability to safely consume an adequate volume.

The effect of an enteral formula on appetite has important consequences for the dietary management of patients receiving nutritional support. For example, in patients also consuming oral diet, the enteral formula should have a minimal effect on appetite in order to prevent the nutrients it provides from displacing those from food. In contrast, enteral formula should be able to satisfy appetite in patients receiving it as a sole source of nutrition, in order to limit the potential for distressing appetite sensations that have previously been reported in patients receiving artificial nutritional support (Padilla et al. 1979; Stratton, 2005).

The effect of enteral formula on appetite has previously been studied in a series of experiments in animals, healthy subjects and patients, although largely in relation to ETF as opposed to oral consumption (Stratton \& Elia, 1999). ETF has been reported to have no effect on appetite and little effect on energy intake from food (Stratton et al. 1998), irrespective of whether it is infused during the day, during the night or continuously for $24 \mathrm{~h}$ (Stratton et al. 2003). This lack of effect on appetite may in part be due to a reduction in cephalic response due to bypass of the upper gastrointestinal tract (Stratton et al. 2003).

Despite much research in ETF there has been little investigation of the effect of oral consumption of enteral formula on appetite. Any effect on appetite is likely to depend upon the composition of the enteral formula, which is under constant development. For example, although standard formulas are devoid of all non-digestible carbohydrate, formulas supplemented with NSP such as pea-fibre and fructo-oligosaccharides (FOS) have been developed in order to improve gastrointestinal function (Wolf et al. 2003). However, despite 
the importance to the dietary management of patients receiving nutritional support, the effect on appetite of a standard enteral formula and one containing such NSP has not been investigated (Stratton \& Elia, 1999). The aim of the present study was to compare appetite within healthy subjects consuming both a standard formula and one supplemented with pea-fibre and FOS as a sole source of nutrition.

\section{Methods}

Subjects

Healthy subjects aged between 21 and 34 years were recruited to a prospective, randomised, double-blind, cross-over trial. Exclusion criteria were: gastrointestinal disorders; diabetes; chronic viral or inflammatory disorders; any prescription medication in the previous month (except for the contraceptive pill); intolerance to FOS; BMI of $<20$ or $>30 \mathrm{~kg} / \mathrm{m}^{2}$; self-reported eating disorders; or currently following a weight-reducing diet. Recruitment was via circular email of all staff and postgraduate students at King's College London. None of the subjects had previously consumed enteral formula but were allowed to taste the study formula prior to recruitment in order for them to judge whether they would be able to comply with consuming it as a sole source of nutrition. Subjects were compensated for taking part in the study. Written informed consent was obtained from each subject and the study was approved by the King's College London Research Ethics Committee.

\section{Protocol}

Subjects consumed enteral formula as a sole source of nutrition for two $14 \mathrm{~d}$ periods that were separated by a 6-week washout phase of normal diet. During these two enteral formula periods, subjects consumed either a standard formula (Nutren 1.0, Nestlé, Konelfingen, Switzerland) or one supplemented with pea-fibre and FOS (Nutren fiber, Nestlé) in random order. During enteral formula periods no other dietary intake was permitted except for water ad libitum and a maximum of three cups of black tea or black coffee per day to prevent caffeine withdrawal and subsequent attrition of subjects (Silk et al. 2001). Random assignment was conducted using a computerised random allocation program (Epistat, Texas, USA), with both subjects and researchers blind to enteral formula allocation. Subjects were weighed at the start and at the end of each enteral formula period.

\section{Enteral formulas}

The volume of enteral formula prescribed was based upon calculated total energy expenditure and rounded to the nearest $250 \mathrm{ml}$ for convenience. Total energy expenditure was calculated by adjusting BMR (calculated using modified Schofield equations) for occupational and non-occupational activity using standard physical activity level tables, in line with normal clinical practice (Department of Health, 1991). The prescription of enteral formula was sufficient to achieve reference nutrient intakes for all vitamins and minerals (Department of Health, 1991). Both enteral formulas were vanilla flavoured and additional flavour sachets (devoid of NSP) were also provided to improve palatability (Moody \& Mayberry, 1998), although their use and their effect on formula palatability was not recorded. To further mimic the clinical scenario, subjects were advised to consume their prescribed volume of formula throughout the day, but were free to determine the exact pattern of consumption. Subjects consumed the formula from a $250 \mathrm{ml}$ cup and recorded their intake in a compliance diary. Where they could not consume the entire $250 \mathrm{ml}$ cup, they estimated and recorded the proportion actually consumed. Compliance with prescription was calculated as the percentage of enteral formula consumed $v$. prescribed. Subjects also recorded any food intake in the compliance diary.

The two enteral formulas were virtually identical in nutrient composition except for the content of pea-fibre and FOS (Table 1). The pea-fibre/FOS formula contained pea-fibre $10 \mathrm{~g} / \mathrm{l}$, providing approximately $50 \%$ fermentable and $50 \%$ non-fermentable fractions, and short-chain FOS (5 g/l). Enteral formulas were provided in identical coded tins to 'blind' both subjects and the researchers from the allocation.

\section{Appetite ratings}

Subjects recorded appetite on $4 \mathrm{~d}$ during consumption of both the standard and the pea-fibre/FOS formula (days 1, 4, 8, 11 and days $57,60,64,67)$. Therefore, appetite was recorded on the same days of the week for each subject in order to allow direct comparison between enteral formulas. Appetite was also recorded on $4 \mathrm{~d}$ during normal diet, however statistical comparison could not be conducted as only the order of the two enteral formula periods had been randomised.

Appetite was recorded hourly from 08.00 to 21.00 hours on standard $100 \mathrm{~mm}$ lines anchored at each end by a phrase denoting the most extreme appetite sensation (Stubbs et al. 2000). The six appetite ratings were hunger $(0=$ not at all hungry, $100=$ as hungry as I've ever felt); fullness $(0=$ not at all full, $100=$ as full as I've ever felt); satiety $(0=$ completely empty, $100=$ cannot eat another bite $)$; desire to eat $(0=$ very weak, $100=$ very strong $)$; prospective consumption $(0=$ nothing at all, $100=$ a large amount); and preoccupation with food $(0=$ no thoughts of food, $100=$ very preoccupied) (Flint $e t$ al . 2000; Stubbs et al. 2000). As subjects were not previously familiar with the use of appetite ratings they received both spoken and written training from a Registered Dietitian.

\section{Statistical analysis}

The distance from $0 \mathrm{~mm}$ recorded on the $100 \mathrm{~mm}$ lines was measured for each hour on each of the $4 \mathrm{~d}$ during consumption

Table 1. Macronutrient and NSP composition of the standard and pea-fibre/fructo-oligosaccharide (FOS) enteral formulas

\begin{tabular}{lcc}
\hline & \multicolumn{2}{c}{ Composition of $1000 \mathrm{ml}$} \\
\cline { 2 - 3 } & Standard formula & Pea-fibre/FOS formula \\
\hline Energy (kJ) & 4184 & 4184 \\
Protein $(\mathrm{g})$ & $40 \cdot 0$ & $40 \cdot 1$ \\
Fat $(\mathrm{g})$ & 38 & 38 \\
Carbohydrate $(\mathrm{g})$ & $126 \cdot 3$ & $126 \cdot 5$ \\
Pea-fibre $(\mathrm{g})$ & 0 & 10 \\
FOS $(\mathrm{g})$ & 0 & 5 \\
\hline
\end{tabular}


of both enteral formulas. Mean, maximum and minimum daily appetite ratings were calculated and compared between enteral formulas using paired $t$ tests. Mean hourly appetite ratings were calculated and compared between enteral formulas using a repeated measures ANOVA. All other mean values (e.g. formula intake, compliance, weight loss and nutrient intake) were compared between enteral formula periods using paired $t$ tests. A value of $P<0.05$ was considered to be statistically significant. Data are presented as means and $95 \%$ CI unless otherwise stated. All statistical analysis was conducted using SPSS for Windows, version 11.0 (SPSS Inc., Chicago, IL, USA).

\section{Results}

Fourteen healthy subjects (nine females, five males) were recruited to the study. During the first enteral formula period two female subjects, who were unable to consume more than $50 \%$ of their calculated energy expenditure due to their dislike of the formula, dropped out of the study after $2 \mathrm{~d}$. Another female subject dropped out for personal reasons unrelated to the study. Thus, eleven healthy subjects (five male and six female) with a mean age of 28 years 2 months (range 25 years 6 months to 30 years 10 months) completed the study and were included in the analysis. Their mean weight was 69.2 (range 61.7-76.9) $\mathrm{kg}$, mean height was 1.71 (range $1.66-1.77$ ) $\mathrm{m}$ and mean BMI was 23.5 (range 22.0-25.0) $\mathrm{kg} / \mathrm{m}^{2}$.

\section{Formula intake, compliance and body weight}

The mean volume of enteral formula prescribed was 2272 (95\% CI 2054, 2491) $\mathrm{ml} / \mathrm{d}$. The mean volume actually consumed was $1988(95 \%$ CI 1759, 2216) $\mathrm{ml} / \mathrm{d}$ for the standard formula and $1937(95 \%$ CI 1759, 2114) $\mathrm{ml} / \mathrm{d}$ for the peafibre/FOS formula $(P=0 \cdot 332)$, corresponding to a compliance with prescription of $88(95 \%$ CI 79, 98) \% and 85 (95\% CI 79, 93) $\%$, respectively $(P=0 \cdot 252)$. Compliance with prescription did not differ significantly between the first $(88 \%$; $95 \%$ CI 81, $95)$ and second $(85 \%, 95 \%$ CI 75, 95) enteral formula period $(P=0 \cdot 100)$. Macronutrient intakes did not differ between consumption of standard and pea-fibre/FOS formulas (Table 2). No subject reported consuming food during either enteral formula period.
There were significant changes in body weight throughout the study $(P<0 \cdot 0005)$. This was due to differences in body weight between the start $(69.2 \mathrm{~kg} ; 95 \%$ CI $61.6,76.9)$ and the end $(67.6 \mathrm{~kg} ; 95 \% \mathrm{CI} 60.4,74.9)$ of the first enteral formula period $(P=0.005)$, and between the start $(68.9 \mathrm{~kg} ; 95 \%$ CI $61.1,76.9)$ and the end $(67.6 \mathrm{~kg} ; 95 \%$ CI $60.3,74.9)$ of the second enteral formula period $(P=0 \cdot 025)$. There were no differences in body weight between the start of the study and the end of the 6-week washout phase $(P=1 \cdot 0)$, indicating that subjects returned to baseline weight during the normal diet washout phase.

Despite the changes in body weight during enteral formula periods, there was no significant difference in mean weight loss between consumption of the standard formula $(1.3 \mathrm{~kg}$; $95 \%$ CI $0.4,2.3)$ and pea-fibre/FOS formula $(1.6 \mathrm{~kg} ; 95 \%$ CI $1 \cdot 0,2 \cdot 3)(P=0 \cdot 388)$. Weight loss was negatively correlated with compliance with formula prescription (Pearson's correlation coefficient $-0.718, P<0.0005)$. Therefore, poorer compliance (lower percentage intake of formula) resulted in greater weight loss, suggesting that subjects were not covertly consuming food during the enteral formula periods.

\section{Appetite ratings}

Mean, maximum and minimum daily appetite ratings were calculated and compared between standard formula and peafibre/FOS formula (Table 3). No order effects were detected for any of the appetite ratings.

Consumption of the pea-fibre/FOS formula resulted in higher mean fullness (46 v. $37 \mathrm{~mm}, P=0.035)$, minimum fullness $(13 v .9 \mathrm{~mm}, P=0.024)$ and minimum satiety $(12 v .8 \mathrm{~mm}$, $P=0.012)$ compared to the standard formula. The lower maximum prospective consumption during the pea-fibre/FOS formula did not reach statistical significance $(P=0.065)$. There were no other statistically significant differences in appetite ratings between formulas. Mean daily appetite ratings during normal diet were: hunger $39 \mathrm{~mm}$ (95\% CI 33, 46); fullness $44 \mathrm{~mm}(95 \%$ CI 37,52$)$; satiety $45 \mathrm{~mm}$ (95\% CI 38, 52); desire to eat $43 \mathrm{~mm}$ (95\% CI 37, 49); prospective consumption $52 \mathrm{~mm}(95 \% \mathrm{CI} 42,62)$; and preoccupation with food $32 \mathrm{~mm}(95 \%$ CI 25,40$)$.

The mean hourly appetite ratings were plotted for the standard and the pea-fibre/FOS formula (Fig. 1). Mean hourly appetite ratings varied throughout the day with statistically significant time effects for hunger $(P=0 \cdot 019)$, fullness

Table 2. Macronutrient, pea-fibre and fructo-oligosaccharides (FOS) intakes of eleven healthy subjects during consumption of standard formula and pea-fibre/FOS formula as a sole source of nutrition*

\begin{tabular}{|c|c|c|c|c|c|}
\hline & \multicolumn{2}{|c|}{ Standard formula } & \multicolumn{2}{|c|}{ Pea-fibre/FOS formula } & \multirow[b]{2}{*}{$P$ value } \\
\hline & Mean & $95 \% \mathrm{Cl}$ & Mean & $95 \% \mathrm{Cl}$ & \\
\hline Energy (kJ) & 8316 & 7361,9271 & 8103 & 7359,8846 & 0.332 \\
\hline Protein (g) & 79.5 & $70 \cdot 4,88 \cdot 6$ & 77.7 & $70 \cdot 6,84 \cdot 8$ & 0.385 \\
\hline Fat $(g)$ & 75.5 & $66 \cdot 9,84 \cdot 2$ & 73.6 & $66 \cdot 8,80 \cdot 4$ & 0.330 \\
\hline Carbohydrate (g) & $251 \cdot 0$ & $222 \cdot 2,279 \cdot 9$ & $245 \cdot 0$ & $222 \cdot 5,267.5$ & 0.361 \\
\hline Pea-fibre (g) & - & & $18 \cdot 3$ & $16 \cdot 8,19 \cdot 9$ & - \\
\hline
\end{tabular}

${ }^{*}$ For details of procedures, see p. 351.

$\dagger P$ value of macronutrient intakes during standard formula $v$. pea-fibre/FOS formula (paired $t$ test). 
( $P=0.028)$, satiety $(P=0.013)$, desire to eat $(P<0.0005)$, prospective consumption $(P=0.007)$ and preoccupation with food $(P<0 \cdot 0005)$. The within-subject effect of enteral formula on mean hourly appetite ratings was significant for fullness $(P=0.037)$, but not for hunger $(P=0.095)$, satiety $(P=0 \cdot 105)$, desire to eat $(P=0 \cdot 210)$, prospective consumption $(P=0.412)$ and preoccupation with food $(P=0.194)$. There were no interactions between time and enteral formula for any of the appetite ratings.

\section{Discussion}

Liquid enteral formulas are commonly used as a sole source of nutritional support of patients in hospital and community settings. Their effect on appetite is potentially important to the dietary management of such patients, however, the effect on appetite of oral consumption of a standard formula and one containing pea-fibre and FOS has not previously been investigated. Therefore the aim of the present study was to compare appetite within healthy subjects consuming both a standard formula and one supplemented with pea-fibre and FOS as a sole source of nutrition.

Subjects consumed a standard and a pea-fibre/FOS formula as a sole source of nutrition for two $14 \mathrm{~d}$ periods. Macronutrient intakes (total energy, protein, fat, carbohydrate) did not differ during these periods (Table 2) and therefore the observed increase in mean and minimum fullness and minimum satiety with the pea-fibre/FOS formula are likely to be the result of differences in formula composition, notably the presence of pea-fibre and FOS.

Fibre has been shown to affect appetite in a variety of studies and via a number of oral, gastric and post-gastric mechanisms. Pea-fibre may have affected the mouthfeel of the formula and therefore contributed to subjective appetite responses. However, subjects did not report differences in mouthfeel between the formulas, perhaps due in part to the 6-week washout period. Fibre may delay gastric emptying thus prolonging gastric distension and appetite signalling (Sturm et al. 2004). However, standard, soya-polysaccharide formula and pea-fibre formulas have recently been shown to have similar gastric emptying rates (Bouin et al. 2001). Post-gastric mechanisms include a potential role for fermentation in appetite signalling. For example, fermentable guar gum may prolong nutrient contact with small intestinal chemoreceptors (French \& Read, 1994) therefore enhancing the production of a range of gastrointestinal hormones (Strader \& Woods, 2005). Research in animal models has shown that fermentable fibres (Massimino et al. 1998) and FOS (Cani et al. 2004; Delzenne et al. 2005) increase the secretion of glucagon-like peptide-1, a hormone known to increase satiety in man. In addition, propionate, a SCFA produced by colonic fermentation of fibre, also increases satiety (Liljeberg et al. 1995). Despite the mechanisms by which fermentable fibres may influence satiety, a human feeding study has demonstrated that non-fermentable fibre has a greater effect on satiety than fermentable fibre (Howarth et al. 2003). Furthermore, the addition of fibre to an enteral formula can reduce the glycaemic response (Hofman et al. 2004), which may independently reduce postprandial appetite (Anderson \& Woodend, 2003). The complex mechanisms by which fibres and FOS affect appetite are yet to be completely elucidated. 

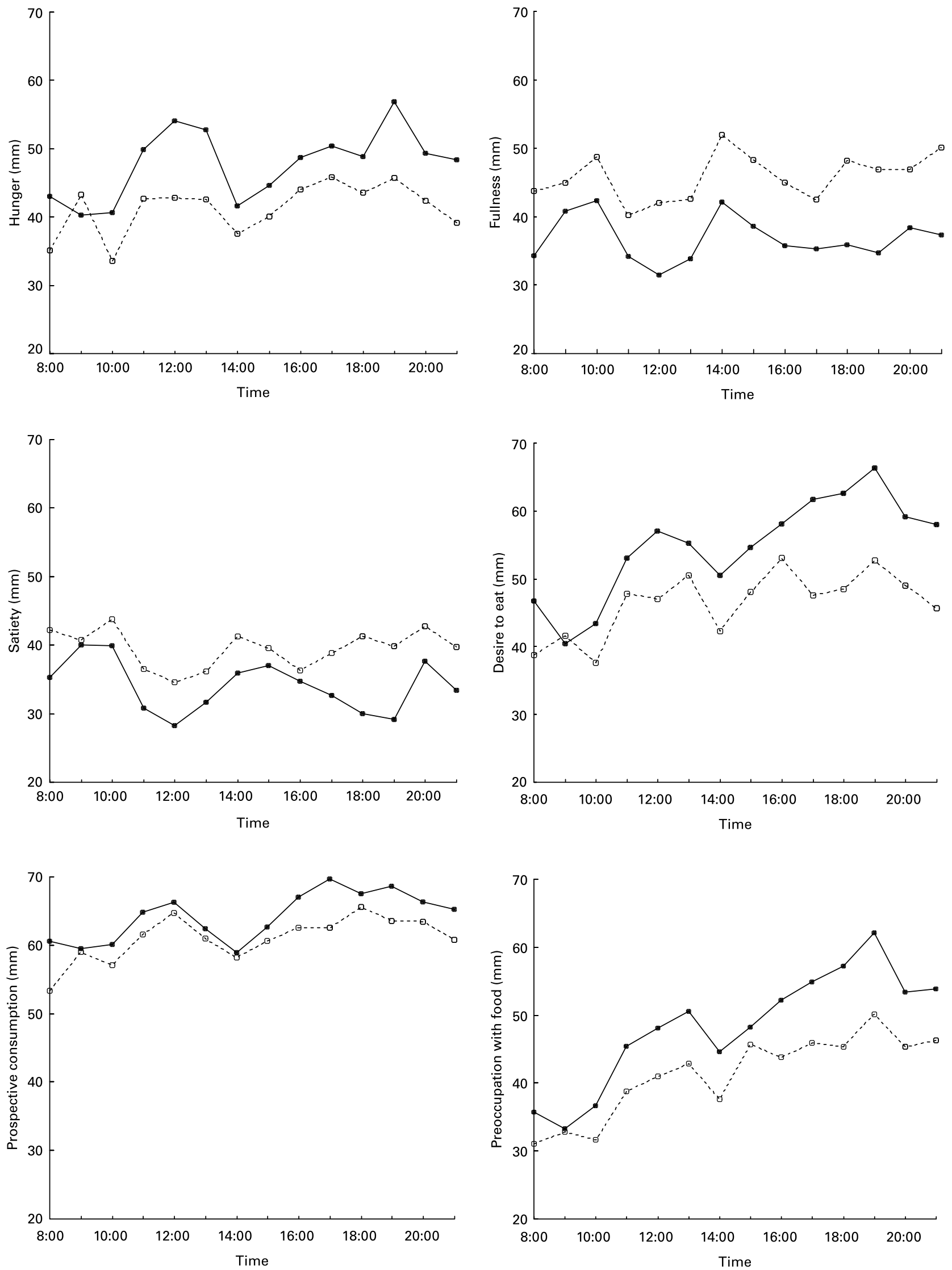

Fig. 1. Mean hourly appetite ratings, recorded on $100 \mathrm{~mm}$ lines, of eleven healthy subjects during consumption of a standard formula ( $\mathbf{\square})$ and a pea-fibre/FOS formula $(\square)$ as a sole source of nutrition. For details of procedures, see p. 351. The within-subject effect of the enteral formula on mean hourly appetite ratings were as follows: hunger $(P=0.095)$; fullness $(P=0.037)$; satiety $(P=0.105)$; desire to eat $(P=0.210)$; prospective consumption $(P=0.412)$; preoccupation with food $(P=0.194)$. 
The findings from the present study offer useful insights into the effect of the composition of an enteral formula on appetite. Extrapolation of the present results to the clinical situation is impeded by inherent differences between healthy subjects and patients consuming enteral formula. These differences include disease, physical activity, drug prescription, age and nutritional status, all of which can affect appetite.

The lower mean and minimum fullness and minimum satiety during consumption of a standard formula would have a number of implications were it to occur in patients requiring nutritional support as a sole source of nutrition. Firstly, these appetite sensations may be distressing for the patient (Padilla et al. 1979; Stratton, 2005), and secondly, they may encourage patients to consume food. Consumption of food could result in a reduction in the efficacy of the enteral formula where it is being used as a primary therapy (e.g. Crohn's disease). However, it is important to note that although the difference in maximum desire to eat approached statistical significance (standard $89 \mathrm{~mm} v$. pea-fibre/FOS $83 \mathrm{~mm}, P=0.095$ ), there was no difference in mean desire to eat (standard $55 \mathrm{~mm}$ v. pea-fibre/FOS $47 \mathrm{~mm}, \quad P=0.233$ ) between formulas. Whether there is a role for pea-fibre/FOS formulas in increasing fullness and reducing supplementary food intake warrants investigation.

Conversely, where patients require enteral formula to supplement an inadequate oral diet (e.g. anorexia, hypermetabolism, undernutrition) there may be a benefit in standard formulas resulting in less fullness compared to a pea-fibre/ FOS formula. Standard formula has been shown to increase nutrient intake without displacing food in community patients receiving oral nutritional support (Stratton, 2000) and healthy subjects receiving ETF (Stratton et al. 2003). Research on the effect on appetite of the composition of the enteral formula when used to supplement oral diet is needed.

The temporal changes in mean hourly appetite demonstrated a largely tri-phasic response throughout the day (Fig. 1). For both enteral formulas the troughs in mean hourly hunger, desire to eat, prospective consumption, preoccupation with food, and peaks in mean hourly fullness and satiety occurred between 9.00 and 10.00 hours, 14.00 and 15.00 hours, and 20.00 and 21.00 hours. These largely reflect the subjects' pattern of consumption of the enteral formulas around normal mealtimes, as noted from compliance records. However, no comparison of appetite $v$. volume of formula consumed could be conducted as the pattern of formula intake varied both throughout the day and between subjects. For this reason mean daily appetite ratings were calculated to account for differences in the pattern of intake, a method frequently used in other studies of appetite in free-living subjects (King et al. 1997; Stubbs et al. 2004).

\section{Methodological limitations and future research}

The present study was conducted under free-living conditions in order to mimic clinical practice. However, this inherently introduces experimental variability, such as the volume and timing of enteral formula or water consumption. For example, in general, subjects did not consume the total daily prescribed volume of enteral formula. This has also been reported in patients consuming enteral formula as a sole source of nutrition (Teahon et al. 1995) and is likely to be due to both sensory-specific satiety (Bell et al. 2003) and the absence of dietary variety (Sørensen et al. 2003). This under-compliance is the likely cause of the weight loss observed during both enteral formula periods. Other experimental variability included the subjects' environment and occupational and non-occupational activities, all of which can result in inter-diurnal variations in appetite (Womble et al. 2003). In order to minimise this experimental variability, appetite was recorded on the same day of the week for each enteral formula period. Investigation of the present findings under experimental conditions in the laboratory would control for this variability, but may reduce its applicability to the clinical setting.

Rating appetite on $100 \mathrm{~mm}$ lines is a valid and reliable indicator of appetite under both experimental and free-living conditions, particularly when using a within-subject design, as in the current study (Stubbs et al. 2000). However, in free-living subjects it elicits results that correlate with subsequent food intake, but not actual nutrient intake (Stubbs et al. 2000). It is therefore important to consider that although statistical differences in fullness and satiety were demonstrated in the present study, their clinical significance (e.g. subsequent nutrient intake) is unclear. Another method for quantifying appetite under free-living conditions is the use of biomarkers such as glucagon-like peptide-1 and ghrelin (De Graaf et al. 2004). Serum glucagon-like peptide-1 is a sensitive measure of changes in satiety, whilst ghrelin, the endogenous ligand for the growth hormone secretagogue receptor, strongly correlates with a range of appetite measures (Strader \& Woods, 2005). Future research on the composition and delivery of enteral formulas should consider the measurement of such appetite biomarkers.

The results presented in the current study offer an interesting insight into the effect on appetite of consuming standard and pea-fibre/FOS enteral formulas as a sole source of nutrition. Future research on the effect on appetite of both the delivery schedule (i.e. supplementary $v$. sole source of nutrition) and the delivery method (i.e. oral $v$. ETF) of standard and peafibre/FOS formulas is required. In addition, comparing appetite when changing from normal diet to consumption of enteral formula would elicit interesting information regarding the effect of changes in physical (i.e. solid to liquid), sensory and nutritional properties of the diet on appetite. These studies should be conducted in both healthy volunteers and in patients requiring nutritional support in order to inform evidence-based clinical nutrition practice. Elucidating the mechanisms through which the composition of the enteral formula affect appetite will contribute to the clinically important issue of maximising nutrient intake in patients receiving nutritional support.

\section{Conclusion}

This is the first study to compare the effect of a standard and a pea-fibre/FOS formula on appetite. Consuming an enteral formula supplemented with pea-fibre and FOS as a sole source of nutrition increases fullness compared to a standard formula. The results will inform future research regarding appetite modulation of patients receiving nutritional support through alteration in the composition of the enteral formula. 


\section{Acknowledgements}

This research was jointly supported by King's College London and Nestlé UK. The enteral formulas were a kind gift from Nestlé Switzerland. The authors would like to thank the subjects for their dedication throughout the study.

\section{References}

Anderson GH \& Woodend D (2003) Effect of glycemic carbohydrates on short-term satiety and food intake. Nutr Rev 61, S17-S26.

Bell EA, Roe LS \& Rolls BJ (2003) Sensory-specific satiety is affected more by volume than by energy content of a liquid food. Physiol Behav 78, 593-600.

Bouin M, Savoye G, Herve S, Hellot MF, Denis P \& Ducrotte P (2001) Does the supplementation of the formula with fibre increase the risk of gastro-oesophageal reflux during enteral nutrition? A human study. Clin Nutr 20, 307-312.

Cani PD, Dewever C \& Delzenne NM (2004) Inulin-type fructans modulate gastrointestinal peptides involved in appetite regulation (glucagon-like peptide-1 and ghrelin) in rats. Br J Nutr 92, $521-526$.

De Graaf C, Blom WA, Smeets PA, Stafleu A \& Hendriks HF (2004) Biomarkers of satiation and satiety. Am J Clin Nutr 79, 946-961.

Delzenne NM, Cani PD, Daubioul C \& Neyrinck AM (2005) Impact of inulin and oligofructose on gastrointestinal peptides. Br J Nutr 93, S157-S161.

Department of Health (1991) Dietary Reference Values for Food Energy and Nutrients for the United Kingdom. London: HMSO.

Flint A, Raben A, Blundell JE \& Astrup A (2000) Reproducibility, power and validity of visual analogue scales in assessment of appetite sensations in single test meal studies. Int J Obes Relat Metab Disord 24, 38-48.

French SJ \& Read NW (1994) Effect of guar gum on hunger and satiety after meals of differing fat content: relationship with gastric emptying. Am J Clin Nutr 59, 87-91.

Hofman Z, van Drunen JD, de Later C \& Kuipers H (2004) The effect of different nutritional feeds on the postprandial glucose response in healthy volunteers and patients with type II diabetes. Eur $J$ Clin Nutr 58, 1553-1556.

Howarth NC, Saltzman E, McCrory MA, Greenberg AS, Dwyer J, Ausman L, Kramer DG \& Roberts SB (2003) Fermentable and nonfermentable fiber supplements did not alter hunger, satiety or body weight in a pilot study of men and women consuming selfselected diets. $J$ Nutr 133, 3141-3144.

King NA, Lluch A, Stubbs RJ \& Blundell JE (1997) High dose exercise does not increase hunger or energy intake in free living males. Eur J Clin Nutr 51, 478-483.

Liljeberg HG, Lonner CH \& Bjorck IM (1995) Sourdough fermentation or addition of organic acids or corresponding alts to bread improves nutritional properties of starch in healthy humans. $J$ Nutr 125, 1503-1511.

Massimino SP, McBurney MI, Field CJ, Thomson AB, Keelan M, Hayek MG \& Sunvold GD (1998) Fermentable dietary fiber increases GLP-1 secretion and improves glucose homeostasis despite increased intestinal glucose transport capacity in healthy dogs. J Nutr 128, 1786-1793.
Moody GA \& Mayberry JF (1998) Can flavourings improve the palatability of a predigested diet? What patients with inflammatory bowel disease think: a short communication. Ital J Gastroenterol Hepatol 30, 237.

Padilla GV, Grant M, Wong H, Hansen BW, Hanson RL, Bergstrom N \& Kubo WR (1979) Subjective distresses of nasogastric tube feeding. J Parenter Enteral Nutr 3, 53-57.

Pearce CB \& Duncan HD (2002) Enteral feeding. Nasogastric, nasojejunal, percutaneous endoscopic gastrostomy, or jejunostomy: its indications and limitations. Postgrad Med J 78, 198-204.

Silk DBA, Walters ER, Duncan HD \& Green CJ (2001) The effect of a polymeric enteral formula supplemented with a mixture of six fibres on normal human bowel function and colonic motility. Clin Nutr 20, 49-58.

Sørensen LB, Moller P, Flint A, Martens M \& Raben A (2003) Effect of sensory perception of foods on appetite and food intake: a review of studies on humans. Int J Obes Relat Metab Disord 27, $1152-1166$.

Strader AD \& Woods SC (2005) Gastrointestinal hormones and food intake. Gastroenterology 128, 175-191.

Stratton RJ (2000) Summary of a systematic review on oral nutritional supplement use in the community. Proc Nutr Soc 59, 469-476.

Stratton RJ (2005) Elucidating effective ways to identify and treat malnutrition. Proc Nutr Soc 64, 305-311.

Stratton RJ \& Elia M (1999) The effects of enteral tube feeding and parenteral nutrition on appetite sensations and food intake in health and disease. Clin Nutr 18, 63-70.

Stratton RJ, Stubbs RJ \& Elia M (1998) Interrelationship between circulating leptin concentrations, hunger, and energy intake in healthy subjects receiving tube feeding. $J$ Parenter Enteral Nutr 22, 335-339.

Stratton RJ, Stubbs RJ \& Elia M (2003) Short-term continuous enteral tube feeding schedules did not suppress appetite and food intake in healthy men in a placebo-controlled trial. J Nutr 133, 2570-2576.

Stubbs RJ, Hughes DA, Johnstone AM, Horgan GW, King N \& Blundell JE (2004) A decrease in physical activity affects appetite, energy and nutrient balance in lean men feeding ad libitum. Am J Clin Nutr 79, 62-69.

Stubbs RJ, Hughes DA, Johnstone AM, Rowley E, Reid C, Elia M, Stratton R, Delargy H, King N \& Blundell JE (2000) The use of visual analogue scales to assess motivation to eat in human subjects: a review of their reliability and validity with an evaluation of new hand-held computerized systems for temporal tracking of appetite ratings. $B r J$ Nutr 84, 405-415.

Sturm K, Parker B, Wishart J, Feinle-Bisset C, Jones KL, Chapman I \& Horowitz M (2004) Energy intake and appetite are related to antral area in healthy young and older subjects. Am J Clin Nutr 80, 656-667.

Teahon K, Pearson M, Levi AJ \& Bjarnason I (1995) Practical aspects of enteral nutrition in the management of Crohn's disease. $J$ Parenter Enteral Nutr 19, 365-368.

Wolf BW, Chow J, Snowden MK \& Garleb KA (2003) Medical foods and fructo-oligosaccharides: a novel fermentable dietary fibre. In Oligosaccharides in Food and Agriculture, pp. 118-134 [G Eggleston and GL Coté, editors]. Washington, DC: American Chemical Society.

Womble LG, Wadden TA, Chandler JM \& Martin AR (2003) Agreement between weekly vs. daily assessment of appetite. Appetite $\mathbf{4 0}$, $131-135$. 